\title{
The Inverse Scattering Transformation in the Angular Momentum Plane
}

\author{
H. J. de Vega
}

Laboratoire de Physique Théorique et Hautes Energies`, F-75230 Paris, ${ }^{\star \star}$ France

\begin{abstract}
The inverse scattering transformation (IST) with the angular momentum $(\lambda)$ as a spectral variable turned out to be a useful method to deal with rotationally invariant problems in field theory at higher spatial dimensions [Refs. (1) and (2)]. We derive the direct and inverse scattering problems for the $v$-dimensional radial Schrödinger equation for variable $\lambda$ and fixed energy (negative or positive). We determine the scattering data (SD) in one to one correspondence with the potential and derive the corresponding GelfandLevitan equation. A family of exactly solvable potentials for any $\lambda$ and fixed energy is obtained. The trace identities associated to this IST are derived for both signs of the energy. They relate integrals of local polynomials in the potential and its derivatives times $r^{2 n-1}(n \geqq 1)$ with the SD. The presence of this power of $r$ makes these relations useful in higher dimensions.
\end{abstract}

\section{Introduction}

The applications of the inverse scattering transformation (IST) are mainly limited up to now to non-linear equations in one spatial dimension. We present here an IST to deal with rotationally invariant problems in a number of spatial dimensions $(v)$ higher than one.

We consider a linear eigenvalue equation (the radial Schrödinger equation in the present paper) where the spectral parameter $(\lambda)$ is the angular momentum. It can be written as

$$
\left[-\frac{d^{2}}{d r^{2}}-\frac{1}{4 r^{2}}+v(r)-E\right] \Psi(r, \lambda)=-\frac{\lambda^{2}}{r^{2}} \Psi(r, \lambda) .
$$

We shall choose length units such that $|E|=1$, i.e. we work at fixed "energy".

Our interest in IST in the angular momentum is motivated by quantum field theory. The scattering data (SD) of this IST are natural variables to express re-

$\star$ Laboratoire associé au CNRS

$\star \star$ Postal address : Université Pierre et Marie Curie, Tour 16, $1^{\text {er }}$ etage, 4 Place Jussieu, 75230 Paris Cédex 05, France 
normalized functional determinants like

$$
S_{\text {eff }}=\log \operatorname{Det}\left(\frac{-\nabla^{2}-E+v(\cdot)}{-\nabla^{2}-E}\right) \text { - counterterms }
$$

for rotationally invariant $v(r)$ with $v(\infty)=0$. This kind of determinant appears in the effective action of scalar field theories at the one-loop level. The IST in the angular momentum is a powerful method to find saddle points as well as to study the strong field behavior of $S_{\text {eff }}$. This method allowed us to investigate the large order behavior of the $1 / N$ series in the two-dimensional non-linear sigma model [1] and in the $\left(\vec{\phi}^{2}\right)_{4}^{2}$ model [2]. In these models the $1 / N$ perturbation theory follows from an effective action with the form of Eq. (1.2).

Euclidean QFT corresponds to negative $E$ in Eq. (1.1). Positive $E$ is found for Minkowskian QFT when $v$ is time independent.

In the present paper we develop the direct and inverse scattering problems for Eq. (1.1) when $E=-1$. The case $E=+1$ was considered by several authors $[3,4]$. The linear problems for $E=-1$ and +1 are very different. For $E=-1(+1)$ waves income and outgo from the origin (the infinity). In Sect. II we introduce the Jost solutions and Jost functions for $E=-1$ and we determine the spectrum, the orthogonality and the completeness relations. In Sect. III the Gelfand-Levitan equation for this problem is derived and the scattering data (SD) in one to one correspondence with $v(r)$ are determined. A family of exactly solvable potentials for any $\lambda$ and fixed $E$ is constructed from separable Gelfand-Levitan kernels.

We derive in Sect. IV the trace identities associated to the IST. They are an infinite sequence of equations relating integrals of local polynomials in $v(r)$ and its derivatives, times $r^{2 n-1}(n=1,2,3, \ldots)$ with the scattering data (SD). The presence of this power of $r$ makes these relations useful in higher dimensional problems. The name "trace-identity" is justified because one finds in the $n$th equation the sum of the eigenvalues to the $n$th power plus the $n$th momentum of the continuous SD.

In Sect. V, we summarize the IST for $E=+1$ and we give the corresponding trace identities. In this case there is an infinite number of discrete eigenvalues and we find the zeta function associated to them in the trace identities [Eqs. (5.13-14)].

The IST presented here can be easily generalized to Dirac-type equations with the angular momentum as a spectral parameter. In this case, integrable non-linear systems are generated. This seems not to be the case for a second order linear equation like $(1.1)$ in the interval $(0, \infty)$. We shall return elsewhere to those integrable non-linear systems which are rotationally invariant restrictions of higher dimensional non-linear systems.

\section{The Schrödinger Equation at Fixed Negative Energy and Variable Angular Momentum}

The radial Schrödinger equation in $v$-dimensional space for fixed negative energy $(-1)$ reads

$$
\left[-\frac{d^{2}}{d r^{2}}-\frac{v-1}{r} \frac{d}{d r}+v(r)+1+\frac{l(l+v-2)}{r^{2}}\right] \Psi_{v}(r, l)=0 .
$$


The dependence on $v$ and $l$ can be easily simplified by the introduction of

$$
\begin{aligned}
\chi(r, \lambda) & \equiv r^{(v-1) / 2} \Psi_{\nu}(r, l) \\
\lambda & \equiv l+\frac{v}{2}-1 .
\end{aligned}
$$

Then, one gets

$$
\left[-\frac{d^{2}}{d r^{2}}+v(r)+1+\frac{\lambda^{2}-1 / 4}{r^{2}}\right] \chi(r, \lambda)=0 .
$$

Let us now define a regular solution by the boundary at $r=\infty$

$$
\lim _{r \rightarrow \infty} e^{r} \varphi(r, \lambda)=1 \text {. }
$$

$\varphi(r, \lambda)$ is an entire function of $\lambda^{2}$ because the boundary condition is independent of $\lambda$. It is convenient to introduce Jost-type solutions by the boundary condition at $r=0$

$$
\lim _{r \rightarrow 0} r^{-\lambda-1 / 2} f(r, \lambda)=1
$$

The scalar product associated to the Schrödinger Eq. (4) is

$$
\left(\chi_{1}, \chi_{2}\right)=\int_{0}^{\infty} \frac{d r}{r^{2}} \chi_{1}(r)^{*} \chi_{2}(r)
$$

We shall assume that

$$
\int_{0}^{\infty} r|v(r)| d r<\infty
$$

and also that

$$
v(r) \underset{r \rightarrow 0}{=} O\left(r^{-2+2 \varepsilon}\right), \quad \varepsilon>0 .
$$

It can be easily shown that $f(r, \lambda)$ is analytic in $\lambda$ if $\operatorname{Re}(\lambda)>-\min (\varepsilon, 1)$. The two solutions $f(r, \lambda)$ and $f(r,-\lambda)$ being linearly independent, we can write $\varphi(r, \lambda)$ as

$$
\varphi(r, \lambda)=\frac{1}{\lambda}[F(\lambda) f(r,-\lambda)-F(-\lambda) f(r, \lambda)] .
$$

Here $F(\lambda)$ is the Jost function and it is given by the Wronskian

$$
F(\lambda)=\frac{1}{2} W[\varphi(r, \lambda), \quad f(r, \lambda)] .
$$

Another expression for $F(\lambda)$ is

$$
F(\lambda)=\lim _{r \rightarrow \infty} e^{-r} f(r, \lambda) .
$$

It follows that $F(\lambda)$ is analytic for $\operatorname{Re}(\lambda)>-\min (\varepsilon, 1)$ and that

$$
F\left(\lambda^{*}\right)=F(\lambda)^{*}
$$

For the free equation $(v \equiv 0)$ the solutions can be expressed in terms of cylindric 
functions

$$
\begin{aligned}
\varphi_{0}(r, \lambda) & =\sqrt{\frac{2 r}{\pi}} K_{\lambda}(r) \\
f_{0}(r, \lambda) & =2^{\lambda} \Gamma(\lambda+1) \sqrt{r} I_{\lambda}(r) \\
F_{0}(\lambda) & =(2 \pi)^{-(1 / 2)} \quad 2^{\lambda} \Gamma(\lambda+1) .
\end{aligned}
$$

We can write for purely imaginary $\lambda=i \tau$

$$
\frac{F(i \tau)}{F_{0}(i \tau)}=D(\tau) e^{i \delta(\tau)}
$$

where $D(\tau)$ and $\delta(\tau)$ are even and odd real functions of $\tau$ respectively. Then, for small distances

$$
\varphi(r, i \tau)=\sqrt{\frac{2 r}{\tau \operatorname{sh} \pi \tau}} D(\tau) \sin \left[\tau \ln \left(\frac{r}{2}\right)-\delta(\tau)-\operatorname{Arg} \Gamma(1+i \tau)\right]
$$

where $\ln D(\tau)=\delta(\tau)=0 \quad$ if $v \equiv 0$.

We see that $\delta(\tau)$ is the phase-shift between free and interacting wave solutions near $r=0$ and not near infinity. These solutions form the continuous spectrum. The discrete spectrum consists of the normalizable solutions of Eq. (2.3). There is an eigenfunction for each real positive root $\lambda_{k}$

$$
F\left(\lambda_{K}\right)=0, \quad K=1,2, \ldots, M .
$$

It can be shown that all zeros in $\operatorname{Re} \lambda>0$ are simple and real. Eigenfunctions associated to different $\lambda_{K}$ are orthogonal. Continuum solutions $(\lambda=i \tau)$ are orthogonal to the discrete eigenfunctions. The scalar product of a pair of solutions is easily computed from the Wronskian relation

$$
-\frac{d}{d r} W\left[\chi_{1}, \chi_{2}\right]=\left(\lambda_{1}^{2}-\lambda_{2}^{2}\right) \frac{\chi_{1}(r) \chi_{2}(r)}{r^{2}}
$$

In this way for the continuum solutions we get

$$
\left(\varphi(r, i \tau), \varphi\left(r, i \tau^{\prime}\right)\right)=\frac{\pi}{\tau \operatorname{sh} \pi \tau} D(\tau)^{2}\left[\delta\left(\tau-\tau^{\prime}\right)+\delta\left(\tau+\tau^{\prime}\right)\right]
$$

and for the discrete eigenfunctions

$$
\begin{gathered}
\left(\varphi\left(r, \lambda_{m}\right), \varphi\left(r, \lambda_{m}\right)\right)=\delta_{n m} \frac{1}{c_{n}} \\
\frac{1}{c_{n}} \equiv \int_{0}^{\infty} \frac{d r}{r^{2}} \varphi\left(r, \lambda_{n}\right)^{2},
\end{gathered}
$$

This set of solutions is not only orthogonal but complete as it is shown below. The Green's function is defined as the solution of

$$
\left[-\frac{d^{2}}{d r^{2}}+v(r)+1+\frac{\lambda^{2}-1 / 4}{r^{2}}\right] G\left(r, r^{\prime} ; \lambda\right)=\delta\left(r-r^{\prime}\right)
$$


regular both at $r=0$ and $r=\infty$. It can be expressed as

$$
G\left(r, r^{\prime} ; \lambda\right)=\frac{f\left(r_{<}, \lambda\right) \varphi\left(r_{>}, \lambda\right)}{2 F(\lambda)} .
$$

For the free case $(v \equiv 0)$ this gives

$$
G_{0}\left(r, r^{\prime} ; \lambda\right)=\sqrt{r r^{\prime}} I_{\lambda}\left(r_{<}\right) K_{\lambda}\left(r_{>}\right) .
$$

The completeness relation can be derived by general theorems on differential operators [5] or by integrating $G\left(r, r^{\prime} ; \lambda\right)$ over a large closed contour on the halfplane $\operatorname{Re} \lambda>0$. In this way one gets

$$
\begin{aligned}
\int d \lambda \rho(\lambda) \varphi(r, \lambda) \varphi\left(r^{\prime}, \lambda\right) & =\frac{1}{\pi} \int_{0}^{\infty} \frac{\tau \operatorname{sh} \pi \tau}{D(\tau)^{2}} d \tau \varphi(r, i \tau) \varphi\left(r^{\prime}, i \tau\right) \\
+\sum_{K=1}^{M} c_{K} \varphi\left(r, \lambda_{K}\right) \varphi\left(r^{\prime}, \lambda_{K}\right) & =r^{2} \delta\left(r-r^{\prime}\right) .
\end{aligned}
$$

For the free case Eq. (2.23) reduces to the Kantorovich-Lebedev transform [6].

A dispersion relation for $F(\lambda)$ can be derived by considering the logarithm of the function

$$
V(\lambda) \equiv \prod_{K=1}^{M} \frac{\lambda+\lambda_{K}}{\lambda-\lambda_{K}} \cdot \frac{F(\lambda)}{F_{0}(\lambda)}
$$

This function is analytic and non-zero in the right-half plane and $V(\infty)=1$. From a Cauchy type integral of $\log V(\lambda)$ and Eq. (2.13), it follows that

and

$$
\frac{F(\lambda)}{F_{0}(\lambda)}=\prod_{K=1}^{M} \frac{\lambda-\lambda_{K}}{\lambda+\lambda_{K}} \exp \left[\frac{2 \lambda}{\pi} \int_{0}^{\infty} \frac{d \tau}{\lambda^{2}+\tau^{2}} \ln D(\tau)\right]
$$

$$
\frac{F(\lambda)}{F_{0}(\lambda)}=\prod_{K=1}^{M}\left(1-\frac{\lambda_{K}^{2}}{\lambda^{2}}\right) \exp \left[-\frac{2}{\pi} \int_{0}^{\infty} \frac{\tau d \tau}{\lambda^{2}+\tau^{2}} \delta(\tau)\right]
$$

\section{Gelfand-Levitan Equations}

The fundamental object in the theory of Gelfand and Levitan is a triangular kernal $K\left(r, r^{\prime}\right)$ which has the following properties:

a) $1+\hat{K}$ transforms free wave functions into interacting wave functions

b) $\hat{K}$ is independent of the spectral parameter.

We shall first define this kernel for our case, and then derive the relation of $\hat{K}$ with the spectral data (Gelfand-Levitan equation). Finally we give the connection of this kernel with the potential $v(r)$.

We define

$$
K\left(r, r^{\prime}\right)=\int_{0}^{\infty} d \tau \rho_{0}(i \tau) \varphi_{0}\left(r^{\prime}, i \tau\right)\left[\varphi(r, i \tau)-\varphi_{0}(r, i \tau)\right]
$$


where $\rho_{0}(\lambda)$ is the free measure

$$
\rho_{0}(i \tau)=\frac{\tau \operatorname{sh} \pi \tau}{2 \pi}
$$

The integrand in Eq. (3.1) is an entire function of $\lambda^{2}=-\tau^{2}$, so we can express the kernel $K\left(r, r^{\prime}\right)$ as an integral from $-i \infty$ to $+i \infty$ in the $\lambda$-plane and then deform the contour. One proves in this way that

$$
K\left(r, r^{\prime}\right)=0, \quad \text { if } r^{\prime}<r .
$$

This implies with the aid of Eq. (2.15) that the free and interacting regular solutions are connected by a transformation of Volterra type

$$
\varphi(r, \lambda)=(1+\hat{K}) \varphi_{0}(r, \lambda)=\varphi_{0}(r, \lambda)+\int_{0}^{\infty} \frac{d r^{\prime}}{r^{\prime 2}} K\left(r, r^{\prime}\right) \varphi_{0}\left(r^{\prime}, \lambda\right) .
$$

Similarly one can derive a Volterra representation of $\varphi_{0}$ in terms of $\varphi$

$$
\begin{gathered}
\tilde{K}\left(r, r^{\prime}\right)=\int d \lambda \rho(\lambda) \varphi\left(r^{\prime}, \lambda\right)\left[\varphi_{0}(r, \lambda)-\varphi(r, \lambda)\right], \\
\varphi_{0}(r, \lambda)=(1+\hat{\tilde{K}}) \varphi(r, \lambda)=\varphi(r, \lambda)+\int_{r}^{\infty} \frac{d r^{\prime}}{r^{\prime 2}} \tilde{K}\left(r^{\prime}, r\right) \varphi\left(r^{\prime}, \lambda\right) .
\end{gathered}
$$

The Gelfand-Levitan equations follow from Eqs. (3.2)-(3.4) and the completeness relation (2.21). We multiply Eq. (3.4) for $\lambda=i \tau$ by $|F(i \tau)|^{-2} \varphi(r, i \tau)$ and Eq. (3.2) by $|F(i \tau)|^{-2} \varphi_{0}(r, i \tau)$ and we integrate over $\tau^{2}$ from zero to $\infty$. Using Eq. (3.2) for $\lambda=\lambda_{K}$, we obtain the Gelfand-Levitan (GL) equation for the present case

$$
\Omega\left(r, r^{\prime}\right)+K\left(r, r^{\prime}\right)+\int_{r}^{\infty} \frac{d s}{s^{2}} K(r, s) \Omega\left(s, r^{\prime}\right)=0
$$

for $r \leqq r^{\prime}$. Here

$$
\begin{aligned}
\Omega\left(r, r^{\prime}\right)= & \int_{0}^{\infty} \frac{\tau^{2} d \tau}{2 \pi} \varphi_{0}(r, i \tau) \varphi_{0}\left(r^{\prime}, i \tau\right)\left[\frac{1}{|F(i \tau)|^{2}}-\frac{1}{\left|F_{0}(i \tau)\right|^{2}}\right] \\
& +\sum_{K=1}^{M} c_{K} \varphi_{0}\left(r, \lambda_{K}\right) \varphi_{0}\left(r^{\prime}, \lambda_{K}\right) .
\end{aligned}
$$

From Eq. (3.6) we learn which are the spectral data (SD) in the present case

$$
\mathrm{SD}=\left\{D(\tau), \tau \geqq 0 ; \lambda_{K}, C_{K}, K=1,2, \ldots, M\right\} .
$$

We assume that the spectral data are such that

$$
\begin{gathered}
D(\tau)-1=0\left(\tau^{-2}\right) \\
\lambda_{K}>0<c_{K}, \quad K=1, \ldots, M . \infty
\end{gathered}
$$

Equation (3.8) guarantees that Eq. (3.6) is Fredholm type $\left(\operatorname{Tr} \Omega \Omega^{+}<\infty\right)$. Then it follows by the same argument is in [7] that the solution of the GL Eq. (3.6) exists and is unique. In the next section we shall see that Eq. (2.7) guarantees that $D(\tau)$ satisfies Eq. (3.8). 
Let us now derive the expression for $v(r)$ in terms of the kernel $K\left(r, r^{\prime}\right)$ as well as the partial differential equation satisfied by this kernel.

The Schrödinger equation (2.3) can be written as the eigenvalue problem

$$
\hat{L} \chi(r, \lambda)=-\lambda^{2} \chi(r, \lambda)
$$

where $\hat{L}$ is an hermitian operation for the scalar product (2.6)

$$
\begin{aligned}
\hat{L} & =\hat{L}_{0}+r^{2} \hat{v} \\
\hat{L}_{0} & =-r^{2}\left(\frac{d^{2}}{d r^{2}}-1\right)-\frac{1}{4} .
\end{aligned}
$$

The Volterra type operator $(1+\hat{K})$ verifies

$$
\hat{L}(1+\hat{K})=(1+\hat{K}) \hat{L}_{0}
$$

or

$$
r^{2} \hat{v}=\left[\hat{K}, \hat{L}_{0}\right](1+\hat{K})^{-1} .
$$

Projection of this equation shows that if $\hat{v}$ is hermitian it must be local and

$$
v(r)=-\frac{2}{r} \frac{d}{d r}\left[\frac{K(r, r)}{r}\right]
$$

It also follows that

$$
\left[r^{2} \frac{\partial^{2}}{\partial r^{2}}-s^{2} \frac{\partial^{2}}{\partial s^{2}}+s^{2}-r^{2}-s^{2} v(s)\right] K(r, s)=0, \quad s>r .
$$

When the continuum part of the SD is trivial $(D(\tau)=1,0 \leqq \tau<\infty)$ the GelfandLevitan equation admits a closed solution because the kernal $\hat{\Omega}$ becomes degenerate. We find

$$
K\left(r, r^{\prime}\right)=-\frac{2}{\pi} \sqrt{r r^{\prime}} \sum_{1 \leqq i, j \leqq M} c_{i} K_{\lambda_{i}}(r)\left[W_{(r)}^{-1}\right]_{i j} K_{\lambda_{j}}\left(r^{\prime}\right)
$$

where $W(r)$ is an $M$ by $M$ matrix with entries

$$
W_{i j}(r)=\delta_{i j}+\frac{2}{\pi} c_{i} \int_{r}^{\infty} \frac{d s}{s} K_{\lambda_{t}}(s) K_{\lambda_{j}}(s)
$$

The corresponding potential results

$$
v(r)=-2 \nabla^{2} \log \operatorname{det} W(r),
$$

where $\nabla^{2}=\frac{d^{2}}{d r^{2}}+\frac{1}{r} \frac{d}{d r}$. The solution of the Schrödinger equation for this potential and any $\lambda$ follows directly by replacing $K\left(r, r^{\prime}\right)$ given by Eq. (3.12) into Eq. (3.2).

\section{Trace Identities}

Here we shall trace identities associated to the IST in the angular momentum. Our starting point is the relation between the Fredholm determinant of the oper- 
ator (3.10) and the Jost function

$$
\Delta\left(\lambda^{2}\right) \equiv \operatorname{Det}\left(\frac{\hat{L}+\lambda^{2}}{\hat{L}_{0}+\lambda^{2}}\right)=\frac{F(\lambda)}{F_{0}(\lambda)}
$$

(see the Appendix for a proof). This ratio is an analytic function of $\lambda$ for $\operatorname{Re}(\lambda)>$ $-\min (\varepsilon, 1)$ and it equals one at infinity. Hence

$$
\log \Delta\left(\lambda^{2}\right)=\sum_{n=1}^{\infty} \mathscr{C}_{n} \lambda^{-n}
$$

We shall compute the $\mathscr{C}_{n}$ in two ways: firstly in terms of $v(r)$ and secondly from the Jost function in terms of the SD (Eq. 2.24). For the first method we use the relations

$$
\begin{aligned}
& \frac{\partial}{\partial \lambda^{2}} \log \Delta\left(\lambda^{2}\right)=\operatorname{Tr}\left[G(\lambda)-G_{0}(\lambda)\right] \\
& G(\lambda)=G_{0}(\lambda)-G_{0}(\lambda) r_{r}^{2} v G(\lambda) .
\end{aligned}
$$

We get for the lowest order term

$$
\begin{aligned}
-\operatorname{Tr}\left[G_{0}(\lambda) r^{2} v G_{0}(\lambda)\right] & =-\int_{0}^{\infty} \int_{0}^{\infty} \frac{d r_{1} d r_{2}}{r_{1}^{2}} G_{0}\left(r_{1}, r_{2} ; \lambda\right)^{2} v\left(r_{2}\right) \\
& =-\int_{0}^{\infty} r_{2} d r_{2} v\left(r_{2}\right)\left[K_{\lambda}\left(r_{2}\right)^{2} \int_{0}^{r_{2}} \frac{d r_{1}}{r_{1}} I_{\lambda}\left(r_{1}\right)^{2}+I_{\lambda}\left(r_{2}\right)^{2} \int_{r_{2}}^{\infty} \frac{d r_{1}}{r_{1}} K_{\lambda}\left(r_{1}\right)^{2}\right] .
\end{aligned}
$$

Using the large index behavior of the cylindric functions [6], we obtain

$$
\frac{\partial}{\partial \lambda^{2}} \log \Delta\left(\lambda^{2}\right)=-\frac{1}{4 \lambda^{3}} \int_{0}^{\infty} r v(r) d r+O\left(\lambda^{-5}\right) .
$$

Higher order terms can be computed in an analogous way and we find after some work

$$
\begin{aligned}
\log \Delta\left(\lambda^{2}\right)= & \frac{1}{2 \lambda} \int_{0}^{\infty} r v(r) d r-\frac{1}{8 \lambda^{3}} \int_{0}^{\infty} r^{3} d r\left[(v(r)+1)^{2}-1\right]\left(1+\frac{1}{\lambda^{2}}\right) \\
& +\frac{1}{16 \lambda^{5}} \int_{0}^{\infty} r^{5} d r\left[\frac{1}{2}\left(\frac{d v}{d r}\right)^{2}+(v(r)+1)^{3}-1\right]+O\left(\lambda^{-7}\right) .
\end{aligned}
$$

$\mathscr{C}_{2 n}$ vanish for all $n \geqq 1$.

The asymptotic expansion of $\log \Delta\left(\lambda^{2}\right)$ in terms of SD follows starting from the identity [8]

$$
\oint_{\mathscr{C}} \frac{d \lambda}{2 \pi i} \lambda^{2 z} \frac{d}{d \lambda} \log \frac{F(\lambda)}{F_{0}(\lambda)}=-\sum_{K=1}^{M} \lambda_{K}^{2 z}
$$

where $0<\operatorname{Re}(z)<\frac{1}{2}$ and $\mathscr{C}$ is a contour from $-i \infty$ to $+i \infty$ closed by a large semicircle on the right-half plane. We can express the 1.h.s. of Eq. (4.5) in terms of $D(\tau)$ and $\delta(\tau)$ with the result

$$
\cos \pi z \int_{0}^{\infty} d \tau \tau^{2 z-1} \delta(\tau)+\sin \pi z \int_{0}^{\infty} d \tau \tau^{2 z-1} \ln D(\tau)=\frac{\pi}{2 z} \sum_{K} \lambda_{K}^{2 z} .
$$


We obtain, by analytic continuation up to integer and half-integer values of $z$

$$
\begin{gathered}
\sum_{K=1}^{M} \lambda_{K}^{2 n}=\frac{n(-1)^{n+1}}{(2 n-1) !} \frac{2}{\pi} \int_{0}^{\infty} d \tau\left(\tau \frac{d}{d \tau} \tau\right)^{2 n-1} \delta(\tau) \\
\sum_{K=1}^{M} \lambda_{K}^{2 n-1}=-\left(n-\frac{1}{2}\right) \mathscr{C}_{2 n-1}+\frac{(-1)^{n+1}}{(2 n-2) !}(n-1 / 2) \frac{2}{\pi} \int_{0}^{\infty} d \tau\left(\tau \frac{d}{d \tau} \tau\right)^{2 n-2} \ln D(\tau),
\end{gathered}
$$

where $n=1,2, \ldots$ and we have used Eq. (4.2) and [12]. The residue at $z=0$ of Eq. (4.6) gives Levinson's theorem

$$
\delta(0+)=\pi M .
$$

The trace identities follow by expressing the $\mathscr{C}_{2 n-1}$ in Eqs. $(4.7-8)$ in terms of the potential through Eq. (4.4). We get, for the first identities

$$
\begin{gathered}
\int_{0}^{\infty} r v(r) d r=-4 \sum_{K} \lambda_{K}+\frac{4}{\pi} \int_{0}^{\infty} d \tau \ln D(\tau), \\
0=\sum_{K} \lambda_{K}^{2}+\frac{2}{\pi} \int_{0}^{\infty}\left(\tau \frac{d}{d \tau} \tau\right) \delta(\tau), \\
\int_{0}^{\infty} r^{3} d r\left\{[v(r)+1]^{2}-1\right\}=\frac{16}{3} \sum_{K} \lambda_{K}^{3}+\frac{8}{\pi} \int_{0}^{\infty} d \tau\left(\tau \frac{d}{d \tau} \tau\right)^{2} \ln D(\tau), \\
0=\sum_{K} \lambda_{K}^{4}+\frac{1}{3} \frac{2}{\pi} \int_{0}^{\infty} d \tau\left(\tau \frac{d}{d \tau} \tau\right)^{3} \delta(\tau), \\
\int_{0}^{\infty} r^{5} d r\left\{\frac{1}{2}\left(\frac{d v}{d r}\right)^{2}+[v(r)+1]^{3}-1\right\} \\
=32 \sum_{K}\left(\frac{\lambda_{K}^{3}}{3}-\frac{\lambda_{K}^{5}}{5}\right)+\frac{16}{\pi} \int_{0}^{\infty} d \tau\left[\left(\tau \frac{d}{d \tau} \tau\right)^{2}+\frac{1}{12}\left(\tau \frac{d}{d \tau} \tau\right)^{4}\right] \ln D(\tau) .
\end{gathered}
$$

From the dimensionally regularized Schrödinger determinant, it can be shown that $\mathscr{C}_{2 n-1}$ expresses for any $n$ as an integral of $r^{2 n-1}$ times a local polynomial in $v(r)$ and its derivatives. Hence, $\mathscr{C}_{2 n-1}[v(\cdot)]$ corresponds to the integral of rotationally invariant field configurations over a $2 n$-dimensional space.

\section{Variable Angular Momentum at Fixed Positive Energy}

We summarize in this section results on the IST at fixed positive energy and variable angular momentum. The Schrödinger equation reads in that case, after the transformation (2.2)

$$
\left[-\frac{d^{2}}{d r^{2}}+v(r)-1+\frac{\mu^{2}-1 / 4}{r^{2}}\right] \chi(r, \mu)=0 .
$$

In this section we set $\mu \equiv \ell+v / 2-1$.

The regular solution of Eq. (5.1) is defined by the boundary condition at $r=0$

$$
\lim _{r \rightarrow 0} r^{-\mu-1 / 2} \varphi(r, \mu)=1, \quad \operatorname{Re} \mu>0
$$


The $\theta$-solutions $\psi_{\theta}(r, \mu)$ and $\tilde{\psi}_{\theta}(r, \mu)$ are defined by the boundary at $r=\infty$

$$
\lim _{r \rightarrow \infty}\left[\psi_{\theta}(r, \mu)-\cos (r+\theta)\right]=\lim _{r \rightarrow \infty}\left[\tilde{\psi}_{\theta}(r, \mu)-\sin (r+\theta)\right]=0 .
$$

Here $\theta$ is an arbitrary real parameter $0 \leqq \theta<2 \pi$. We can express $\varphi(r, \mu)$ as the linear combination

$$
\varphi(r, \mu)=v_{\theta}(\mu) \tilde{\psi}_{\theta}(r, \mu)+u_{\theta}(\mu) \psi_{\theta}(r, \mu) .
$$

Here $v_{\theta}(\mu)$ is the Jost function. It is analytic for $\operatorname{Re} \mu>-\varepsilon$ and

$$
v_{\theta}(\mu)^{*}=v_{\theta}\left(\mu^{*}\right) \text {. }
$$

The eigenvalues are given by the real zeros of $v_{\theta}(\mu)$ in $\operatorname{Re} \mu>0$ [3]. It can be shown that there are an infinite number, all real and simple

$$
v_{\theta}\left(\mu_{K}\right)=0, \quad K \text { positive integers. }
$$

There is a continuous spectrum for purely imaginary $\mu$.

In the free case $[v(r) \equiv 0]$ we find

$$
\begin{aligned}
& \varphi_{0}(r, \mu)=2^{\mu} \Gamma(\mu+1) \sqrt{r}_{\mu}(r), \\
& v_{\theta}^{0}(\mu)=\pi^{-1 / 2} 2^{\mu+1 / 2} \Gamma(\mu+1) \sin \left[\theta+\frac{\pi}{2}\left(\mu+\frac{1}{2}\right)\right], \\
& \mu_{K, \theta}^{0}=2 K-\frac{1}{2}-\frac{2 \theta}{\pi}, \quad K-\left[\frac{1}{4}+\frac{\theta}{\pi}\right]=1,2,3, \ldots
\end{aligned}
$$

The inverse scattering problem can be formulated for this case in the GelfandLevitan form. For fixed $\theta$ the spectral data are

$$
S D=\left\{\delta_{\theta}(\tau), 0 \leqq \tau<\infty ; \mu_{K}^{\theta}, c_{K}^{\theta}, K \in \mathcal{N}\right\}
$$

Here

$$
\frac{v_{\theta}(i \tau)}{v_{\theta}^{0}(i \tau)}=D_{\theta}(\tau) e^{i \delta_{\theta}(\tau)} \quad \text { and } \quad c_{K}^{\theta} \equiv u_{\theta}\left(\mu_{K}^{\theta}\right) v_{\theta}^{\prime}\left(\mu_{K}^{\theta}\right)
$$

We have derived the trace identities associated to this spectral transformation. As in the negative energy case, the Jost function can be related to the SD and to the Fredholm determinant $\Delta_{\theta}\left(\mu^{2}\right)$ associated to Eq. (5.1).

and

$$
\Delta_{\theta}\left(\mu^{2}\right)=\frac{v_{\theta}(\mu)}{v_{\theta}^{0}(\mu)}=\prod_{K}\left(\frac{1-\frac{\mu_{K}^{\theta 2}}{\mu^{2}}}{1-\frac{\mu_{K, 0}^{\theta 2}}{\mu^{2}}}\right) \exp \left(-\frac{2}{\pi} \int_{0}^{\infty} \frac{\tau \delta_{\theta}(\tau)}{\mu^{2}+\tau^{2}} d \tau\right)
$$

$$
\log \Delta_{\theta}\left(\mu^{2}\right)=\sum_{1}^{\infty} d_{n, \theta} \mu^{-n}
$$

Following the same procedure as in Sect. IV, we find

$$
\zeta_{\theta}(-2 n)=\frac{(-1)^{n+1} n}{(2 n-1) !} \frac{2}{\pi} \int_{0}^{\infty} d \tau\left(\tau \frac{d}{d \tau} \tau\right)^{2 n-1} \delta_{\theta}(\tau), \quad n=1,2,3, \ldots
$$




$$
\begin{gathered}
\zeta_{\theta}(1-2 n)=-\left(n-\frac{1}{2}\right) d_{2 n-1, \theta}+\frac{(-1)^{n+1}}{(2 n-2) !}\left(n-\frac{1}{2}\right) \frac{2}{\pi} \int_{0}^{\infty} d \tau\left(\tau \frac{d}{d \tau} \tau\right)^{2 n-2} \ln D_{\theta}(\tau), \\
\zeta_{\theta}(0)=\frac{1}{\pi} \delta_{\theta}(0+) .
\end{gathered}
$$

Here the zeta function is defined for $\operatorname{Re} z>0$ as

$$
\zeta_{\theta}(z)=\sum_{K=1}^{\infty}\left[\frac{1}{\left(\mu_{K}^{\theta}\right) z}-\frac{1}{\left(\mu_{K, 0}^{\theta}\right) z}\right] .
$$

For $\operatorname{Re} z \leqq 0, \zeta_{\theta}(z)$ is defined by analytic continuation of Eq. (5.13). Equation (5.12) expresses Levinson's theorem for the present case.

The coefficients $d_{n, \theta}$ can be computed in terms of $v(r)$ as the $\mathscr{C}_{n}$ were computed in Sect. IV. In this way we get the trace identities which are formally identical to Eq. (4.10-14) if the following replacements are done

$$
\begin{aligned}
r & \longrightarrow i r \\
v(r) & \longrightarrow-v(r) \\
\sum_{K} \lambda_{K}^{n} & \longrightarrow \zeta_{\theta}(-n) \\
\delta(\tau), D(\tau) & \longrightarrow \delta_{\theta}(\tau), D_{\theta}(\tau)
\end{aligned}
$$

\section{Final Remarks}

The transformation from $v(r)$ to the scattering data (3.7) or (5.7) is a local diffeomorphism. This is easily obtained by computing infinitesimal variations from the Gelfand-Levitan equation. We find for the case $E=-1$

$$
\begin{aligned}
\delta v(r)= & \frac{1}{r} \frac{d}{d r}\left\{\frac { 1 } { r } \left[-\frac{4}{\pi} \int_{0}^{\infty} \frac{\tau \operatorname{sh} \pi \tau}{D(\tau)^{3}} \varphi(r, i \tau)^{2} \delta D(\tau) d \tau\right.\right. \\
& \left.\left.+2 \sum_{K} \varphi_{K}(r)^{2} \delta c_{K}+4 \sum_{K} \varphi_{K}(r) \dot{\varphi}_{K}(r) \delta \lambda_{K}\right]\right\} .
\end{aligned}
$$

The inverse mapping directly follows, multiplying the preceding equation by appropriate wave functions and integrating over $r$, or by perturbation theory from Eq. (2.3). One obtains

$$
\begin{aligned}
\delta \lambda_{K} & =-\frac{c_{K}}{2 \lambda_{K}} \int_{0}^{\infty} \varphi_{K}(r)^{2} \delta v(r) d r \\
\delta D(\tau) & =\frac{1}{2} \int_{0}^{\infty} d r \delta v(r) \varphi(r, i \tau) \operatorname{Re}\left[f(r, i \tau) e^{i \delta(\tau)}\right] .
\end{aligned}
$$

The IST associated to several linear problems on the real axis can be interpretated as a canonical transformation [9]. That is, there exists a symplectic form invariant under the IST. Equivalently, there is a Poisson bracket which remains unchanged under the IST.

This does not seem to be the case for the linear problem of the present paper and more generally for second-order equations in a semi-infinite interval. 
We can define a Poisson bracket $(\mathrm{PB})$ through

$$
\{A, B\}=\frac{1}{2} \int_{0}^{\infty} \frac{d r}{r^{2}}\left[\frac{\delta A}{\delta v(r)} \frac{\overleftrightarrow{d}}{d r} \frac{\delta B}{\delta v(r)}\right]
$$

by analogy with the $\mathrm{KdV}$ equation. Then, canonical coordinates are

$$
\begin{gathered}
Q(r)=r v(r), \quad P(r)=\int_{r}^{\infty} r^{\prime} v\left(r^{\prime}\right) d r^{\prime} \\
\left\{Q\left(r_{1}\right), P\left(r_{2}\right)\right\}=\delta\left(r_{1}-r_{2}\right) .
\end{gathered}
$$

The PB of the Jost function can be computed in a closed form with the aid of Wronskian identities

$$
\left\{F\left(\lambda_{1}\right), F\left(\lambda_{2}\right)\right\}=\frac{1}{4 \lambda_{1} \lambda_{2} \lambda_{2}+\lambda_{1}} F\left(\lambda_{1}\right) F\left(\lambda_{2}\right), \quad \operatorname{Re}\left(\lambda_{1,2}\right)>0 .
$$

The PB for the SD easily follows

$$
\begin{aligned}
\left\{\ln D(\tau), \ln D\left(\tau^{\prime}\right)\right\} & =\frac{1}{2} \frac{P}{\tau^{\prime 2}-\tau^{2}},\left\{\delta(\tau), \delta\left(\tau^{\prime}\right)\right\}=\frac{1 \tau^{2}+\tau^{\prime 2}}{4} P \frac{1}{\tau \tau^{\prime}} P \frac{1}{\tau^{\prime 2}-\tau^{2}} \\
\left\{\ln D(\tau), \delta\left(\tau^{\prime}\right)\right\} & =\frac{\pi}{4 \tau^{\prime}}\left[\delta\left(\tau-\tau^{\prime}\right)+\delta\left(\tau+\tau^{\prime}\right)\right]
\end{aligned}
$$

where $P$ stands for principal value. Apparently, it is not possible to obtain canonical variables from these SD. This can be related to the fact that a non-linear evolution equation is solvable in $(0+\infty)$ when the dispersion relation is even in the eigenvalue [10]. In our case, if we define the time evolution, taking as hamiltonian a linear combination of the $\mathscr{C}_{n}$ and the SD as canonical variables by definition, we find an odd dispersion law because $\mathscr{C}_{2 n}=0$.

As a last remark, we wish to note that the linear problem (1.1) in the interval $(0, \infty)$ can be related to a problem in the whole real axis. If we set

$$
\begin{aligned}
x & \equiv \ln r \\
V(x) & \equiv e^{2 x}[1+v(r)] \\
\psi(x, \lambda) & \equiv e^{-x / 2} \chi(r, \lambda),
\end{aligned}
$$

we get from Eq. (1.1.)

$$
\left[-\frac{d^{2}}{d x^{2}}+V(x)\right] \psi(x, \lambda)=-\lambda^{2} \psi(x, \lambda), \quad-\infty<x<+\infty .
$$

This is a one-dimensional problem with an exponentially rising barrier on the right. On the left side the potential vanishes asymptotically if we assume that Eq. (2.8) holds.

In conclusion, the scattering problem on the angular momentum is equivalent to a one-dimensional problem with $\left(-\lambda^{2}\right)$ as "energy" and the following boundary conditions on the potential

$$
\lim _{x \rightarrow+\infty} e^{-2 x} V(x)=1, \quad \lim _{x \rightarrow-\infty} V(x)=0 .
$$




\section{Appendix}

The connection between the Fredholm determinant and the $S$-matrix can be proved in a general context (see for example [11]). We give here a simple proof. It follows from Eqs. (4.3) and (2.21)

$$
\begin{aligned}
\frac{\partial}{\partial \lambda^{2}} \log \Delta\left(\lambda^{2}\right) & =\int_{0}^{\infty} \frac{d r}{r^{2}}\left[G(r, r ; \lambda)-G_{0}(r, r ; \lambda)\right] \\
& =\lim _{\varepsilon \rightarrow 0^{+}} \lim _{\mu \rightarrow \lambda} \int_{\varepsilon}^{\infty} \frac{d r}{r^{2}}\left[\frac{f(r, \lambda) \varphi(r, \mu)}{2 F(\lambda)}-\frac{f_{0}(r, \lambda) \varphi_{0}(r, \mu)}{2 F_{0}(\lambda)}\right] .
\end{aligned}
$$

This last integral can be performed using Eq. (2.16), with the result

$$
\frac{\partial}{\partial \lambda^{2}} \log \Delta\left(\lambda^{2}\right)=\lim _{\varepsilon \rightarrow 0^{+}} \lim _{\mu \rightarrow \lambda} \frac{\varepsilon^{\lambda-\mu}}{2 \mu(\lambda-\mu)}\left[\frac{F_{0}(\mu)}{F_{0}(\lambda)}-\frac{F(\mu)}{F(\lambda)}\right]=\frac{d}{d \lambda^{2}} \log \frac{F(\lambda)}{F_{0}(\lambda)} .
$$

Noting that both $\Delta\left(\lambda^{2}\right)$ and $F(\lambda) / F_{0}(\lambda)$ tend to one for $\lambda=\infty$, we get the desired Eq. (4.1).

\section{References}

1. de Vega, H. J. : Phys. Lett. B. 98B, 280 (1981)

2. de Vega, H. J. : LPTHE preprint (in preparation)

3. Regge, T. : Nuovo Cimento 14, 951 (1959)

Burdet, G., Giffon, M., Predazzi, E. : Nuovo Cimento 36, 1337 (1965)

Loeffeld, J. J. : Ann. Inst. Henri Poincaré 8, 339 (1968)

4. Chadan, K., Sabatier, P. C. : Inverse Problems in Quantum Scattering Theory. Berlin, Heidelberg, New York : Springer 1977 (Specially chapters XI and XIII and references contained therein)

5. Naimark, M. A. : Linear Differential Operators, Part II. New York : Frederick Ingar 1968

6. Lebedev, N. N. : Special functions and their applications. London : Prentice Hall 1965 Erdélyi et al. : Tables of Integral Transforms, Vol. 2. New York : 1954 McGraw Hill

7. Newton, R. G. : Scattering Theory. New York : McGraw Hill 1966

Faddeev, L. D. : J. Math. Phys. 4, 72 (1963)

8. See for example

Buslaev, V. S., Faddeev, L. D. : Dokl. Akad. Nauk SSSR 132, 13 (1960); Sov. Math. Dokl. 1, $451(1960)$

9. Zakharov, V. E., Faddeev, L. D. : Funkt. Anal. iego Prilozh. 5, 18 (1971) (Funct, Anal. Appl, 5, $280(1971))$

Takhtadzhyan, L. A., Faddeev, L. D. : Teor. Mat. Fiz. 21, 160 (1974) (Theor. Math. Phys. (USSR) 21, 1046(1975))

See also Faddeev, L. D. : In: Solitons, Topics in Current Physics. Bullough, R. M., Caudrey, P. J. (eds.) Berlin, Heidelberg, New York: Springer 1980

10. Ablowitz, M. J., Segur, H. : J. Math. Phys. 16, 1054 (1975)

I am indebted to Prof. Mark Ablowitz for calling my attention on this paper.

11. Birman, M. S., Krein, M. G. : Dokl. Akad. Nauk. SSSR 144, 740 (1962)

12. Gelfand, I. M. Shilov, G. E. : Generalized Functions. New York : Academic Press 1964

Communicated by E. Brézin

Received December 24, 1980 
